\title{
Research Paper \\ Comparison of Effectiveness of Nutrition and C-CRT on Improving Working Memory in Children With ADHD
}

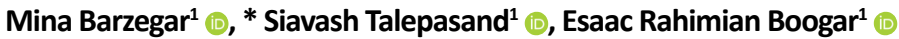

\begin{tabular}{|c|c|}
\hline $\begin{array}{l}\text { Use your device to scan } \\
\text { and read the article online }\end{array}$ & Citation Barzegar M, Talepasand S, Rahimian Boogar E. Comparison of Effectiveness of Nutrition and C-CRT on Improving \\
\hline 口it口 & $\begin{array}{l}\text { Working Memory in Children With ADHD. The Journal of Qazvin University of Medical Sciences. 2019; 22(6):178-189. https://doi. } \\
\text { org/10.32598/JQUMS.22.6.178 }\end{array}$ \\
\hline 口 & doij https://doi.org/10.32598/JQUMS.22.6.178 \\
\hline
\end{tabular}

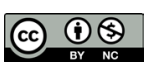

Received: 16 Oct 2018

Accepted: 13 Dec 2018 Available Online: 01 Feb 2019

Keywords:

Attention Deficit Hyperactivity Disorder, Computerized Cognitive Rehabilitation Training, Nutritional supplementations, Working Memory

\begin{abstract}
A B STRACT
Background Improving memory is an indicator of treatment in children with Attention Deficit Hyperactivity Disorder (ADHD).

Objective The present study aimed to compare the effectiveness of Computerized Cognitive Rehabilitation Training (CCRT) , nutrition supplementation intervention, and both combined on the improvement of Working Memory (WM) in children with ADHD.

Methods This was an experimental research with pre-test and post-test, follow-up and control group design. The statistical population included all of the elementary school girls of Tehran City, Iran. Using multilevel clustering, 66 children with ADHD were considered, of whom, 52 were selected for study participation and assigned into 4 experimental and control groups, each consisting of 13 individuals. SNAPIV and clinical interviews were employed to diagnose ADHD. The Raven's Standard Progressive Matrices was conducted to measure the participants' intelligence level. The study participants were matched in age, Intelligence Quotient (IQ), and the gravity of ADHD symptoms. In group one, 22 sessions of 45-minutes of CCRT were performed using Captain's Log software. The second group received zinc, vitamin B6, and omega-3 for 4 months. In the third group, the first phase of the administration of zinc, vitamin B6, and omega-3 supplements was performed; after 15 sessions, CCRT was performed and obtained by the $\mathrm{N}$-back test. Data were analyzed using repeated measures analysis of variance by SPSS.

Findings The obtained results represented the increased mean score of the improvement of WM in the intervention groups, compared to the control group. However, there was no significant difference in WM score between the intervention groups.
\end{abstract}

Conclusion CCRT and nutrition supplementation intervention improve WM in children with ADHD.

\section{Extended Abstract}

\section{Introduction}

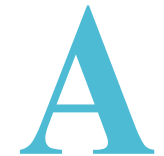

ttention Deficit Hyperactivity Disorder (ADHD) is the most prevalent neurobehavioral disorder among children. The clinical classification of ADHD is based on 6 signs or more of the following symptoms: unrest, the loss of personal belongings, impulsivity, high risk behaviors, excessive speaking, forgetfulness, interrupting conversations, difficulty in understanding homework before the age of 12 years, which lasts for 4 months and will adversely affect the social, occupational, educational and family skills. The symptoms are not better explained by another mental disorder (e.g. mood disorder, anxiety disorder, and substance

\section{* Corresponding Author:}

Siavash Talepasand, PhD.

Address: Department of Educational Sciences, Faculty of Psychology \& Educational Sciences, Semnan University, Semnan, Iran.

Tel: +98 (912) 6040690

E-Mail: stalepasand@semnan.ac.ir 
intoxication). The prevalence of Diagnostic and Statistical Manual of Mental Disorders, $5^{\text {th }}$ Edition (DSM-V) related ADHD disorders is reported to be between $2 \%$ and $21 \%$ and is more prevalent in boys [1].

Barkley's theory focuses on one of the Executive Functions (EF) in general, with a clear application for ADHD. Barkley recognized a failure to inhibit responses as the cause of all other EF deficits observed in ADHD. EF is used to describe higher-order cognitive functions including inhibitory control and Working Memory (WM). WM is referred to the amount of short time memory engagement to support activity and skill $[3,4]$.

The nutritional ADHD evidence suggests that the mammalian brain is rich in unsaturated fatty acids that are not produced in the mammalian body and should be fed through dietary; the role of fatty acids is concerned with fluidity cortex and the function of vector. Among effective nutritional supplements, zinc is involved in the production of fatty acids and serotonin and is a dopamine inhibitor. Evidence suggests that adding omega-3, B vitamins, and iron to children's nutrition program will improve their cognitive functions and reduce ADHDrelated behavioral symptoms $[6,7]$.

There are various treatments available for ADHD, including pharmacotherapy, behavioral therapy, cognitive behavioral therapy, parent-based interventions, Nutritional Therapy (NT), Cognitive Rehabilitation Training (CRT), group therapy and self-esteem enhancement [8]. C-CRT and NT are modern treatments with relatively acceptable empirical support $[11,12]$.

Due to the controversial results from C-CRT and NT studies, which are at a beginning stage, it seems necessary to further explore these therapeutic areas. Therefore, in the present study, in addition to reviewing C-CRT and NT, the effectiveness of combination of these two treatments was investigated on the improvement of WM in children with ADHD [13].

\section{Methods and Materials}

An experimental design with a control group was used with three pre-tests, post-test and follow-up measurements. Raven's Progressive Matrices scores and the severity of disturbances were drawn in a coordinate axis. The students with similar scores of intelligence and the severity of ADHD were selected as quadruple blocks and randomly divided into 4 groups of 13 individuals (three interventional groups and one control group).

Age variables were homogeneous in all groups. In group one, 22 sessions of 45-minutes of C-CRT were performed using Captain's Log software. Zinc, B6 vitamin, and omega-3 were prescribed for 4 months in the second group. In the third group, the first phase of administrating zinc, B6 vitamin and omega-3 supplements was performed, and after 15 sessions, C-CRT sessions were conducted. Data were collected using the N-back test. Data were analyzed using repeated measures Analysis of Variance (ANOVA) by SPSS.

\section{Results}

The obtained results represent the increased mean score on the improvement of WM in the intervention groups in comparison to control group. Although, there was no significant difference in WM scores between the intervention groups. Stability in Precision and promptitude in C-CRT group, NT group, and the combined group was observed in following. Additionally, findings suggest that the effect of time interaction is significant on precision and promptness (Table 1).

\section{Conclusion}

The obtained results indicate that WM in children with ADHD has been promoted in all experimental groups compared to the control group. In C-CRT group, the goal was to reinforce and rehabilitate cognitive components. These exercises provide prompt feedback, sequencing and locative calling, lead to learning new subjects, which improve cognitive abilities and self-control to achieve academic and cognitive success [23]. In addition, consis-

Table 1. The effect of group $\times$ time interaction on the dependent variables

\begin{tabular}{cccccccc}
\hline Variable & Partial Eta Squared & P & F & ms & df & SS & Test Power \\
\hline Precision & 0.429 & 0.0001 & 12.01 & 620.55 & 3 & 3723.33 & 1 \\
Promptness & 0.66 & 0.0001 & 31.07 & 422365.59 & 3 & 1735609.48 & 1 \\
\hline
\end{tabular}


tent with the effectiveness of NT in the present study, the positive effects of omega- 3 have been reported in previous studies. Many studies have revealed the positive effects of vitamins and minerals such as iron and zinc on the growth and performance of neuropsychological skills. In explaining the effectiveness of NT, it can be argued that omega-3, vitamin B6 and zinc play roles in nerve growth and cognitive functions $[24,30]$.

The combined intervention had a significant effect on the improvement of WM, but did not have more efficacy than the other two experimental groups. Of course, the possible explanation is that the time of C-CRT and NT interventions in the combined group, was half the duration of each intervention proprietary, and the short period of time may have reduced the effectiveness of combined intervention. One of the limitations of this study is that the levels of supplementations in the blood of subjects were not measured before the onset of intervention to determine whether they were below the normal levels.

According to this study, it is recommended that C-CRT and NT be used along with pharmacological treatments. It is feasible to perform C-CRT on children. It can even be amusing with the help of family members at home, and may improve attention and WM in them. In addition to improving memory, the use of NT has other advantages, such as strengthening the immune system, promoting health and it is relatively less costly than other treatment methods [31]. Finally, future studies are recommended on investigating the nutritional interventions by measuring the serum levels of these supplementations before and after consumption.

\section{Ethical Considerations}

\section{Compliance with ethical guidelines}

The present research was approved by the Ethics Committee of Semnan University of Medical Sciences (code: IR.SEMUMS.REC.1397.008).

\section{Funding}

The present paper was extracted from the $\mathrm{PhD}$. thesis of the first author, in Department of Educational Sciences, Faculty of Psychology \& Educational Sciences, Semnan University.

\section{Authors' contributions}

All authors contributed in designing, running, and writing all parts of the research.

\section{Conflict of interest}

The authors declared no conflict of interest. 


\title{
مقايسه اثرات درمانى تغذيه و توانبخشى شناختى رايانهاى بر بهبود حافظه كارى كودكان با اختلال نقص توجه/بيش فعالى
}

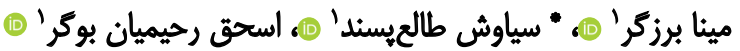

1- كروه روانشناسى تربيتى، دانشكده روانشناسى و علوم تربيتى، دانشكاه سمنان، سمنان، ايران.

\begin{abstract}
يـ
إمينه بهبود حافظه از ويزوكى هاى درمان كودكان مبتّلا به اختلال نقص توجهابيشفعالى است.

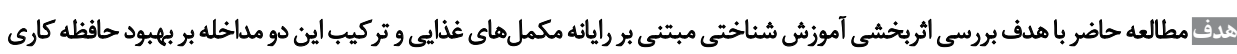

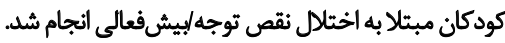

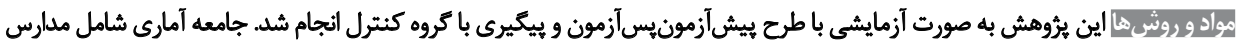

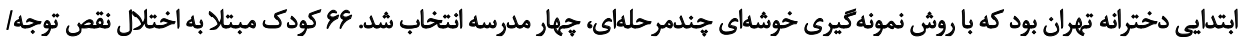

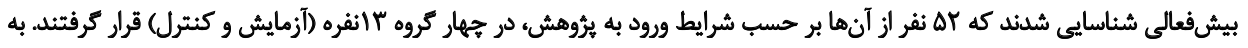

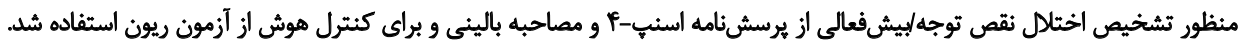

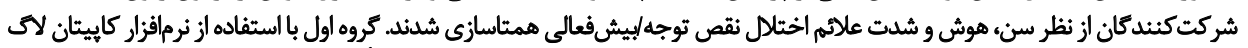

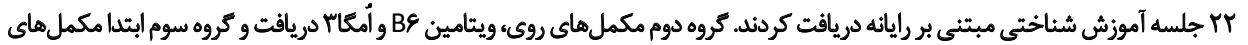

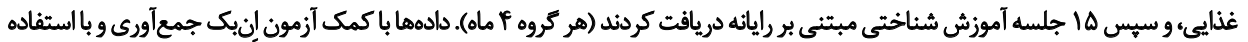

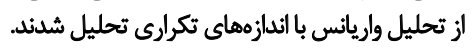

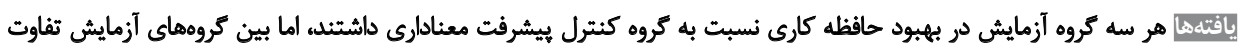

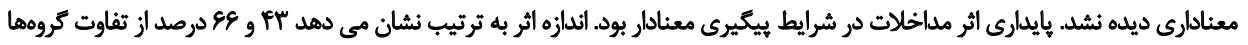

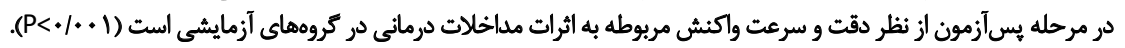

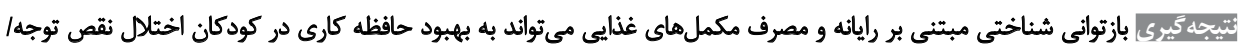
بيشفالى منجر شودي.
\end{abstract}

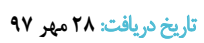

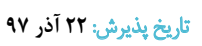

تاريخ اثتشار: I إبهمن

كليدوإوها:

اختثلال نقص توجها

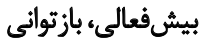
شناختى مبتنى بران

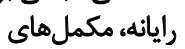
غذايى، حافظه كارى

اثرات منفى بر مهارتهاى اجتماعى، شغلى، تحصيلى وخانوادگى

مقاله

به جاي بكنارد.

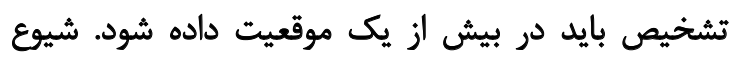

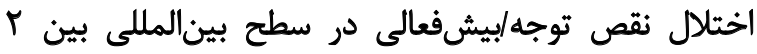

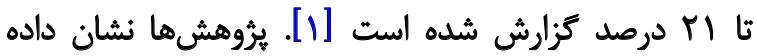

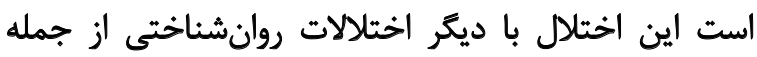

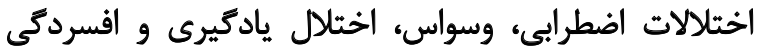

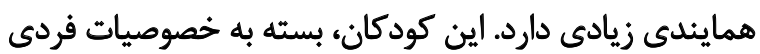

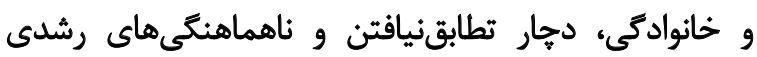

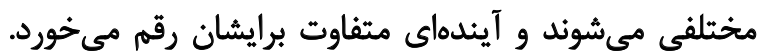

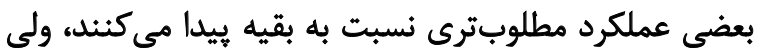
در بعضى ديكر تشخيص اين اختلال در كودكى مي بـ بتواند

اختلال نقص توجه/بيشفعالى' شايعترين اختلال روانيزشكى

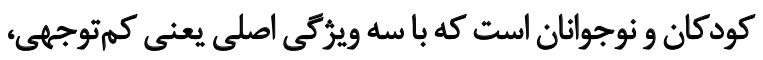

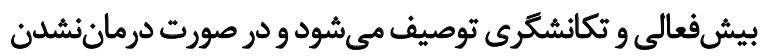

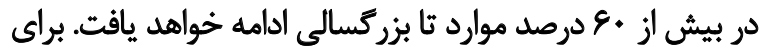

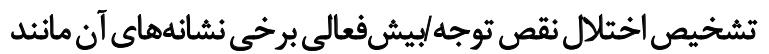

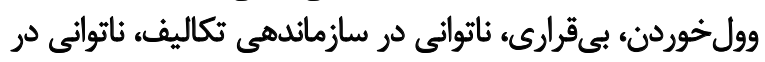

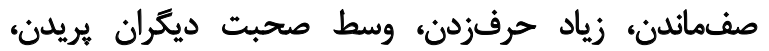

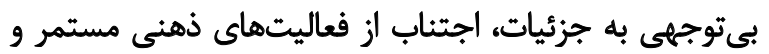

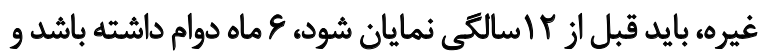

1. Attention Deficit Hyperactivity Disorder (ADHD)

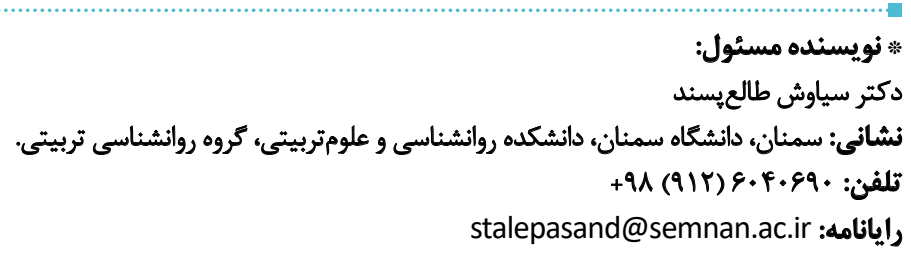


داروها را تحمل كنيند و از سوى ديخًر در بين متخصصان درباره

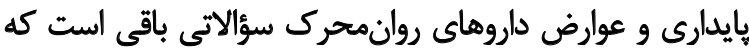

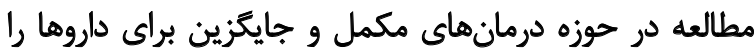

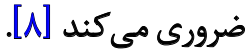

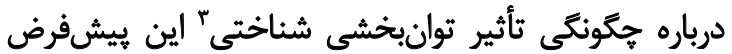

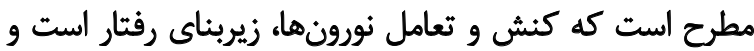

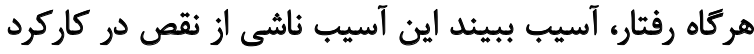

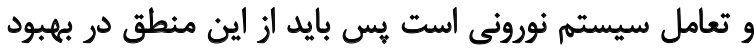

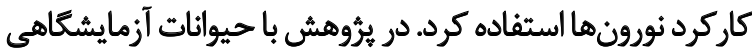

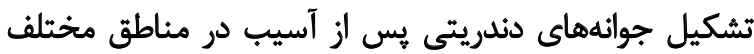

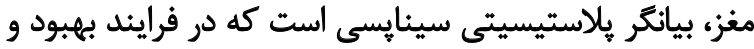

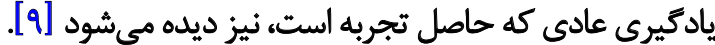
توانبخشى شناختى شامل تمريناتى است كه متمركز بر برات

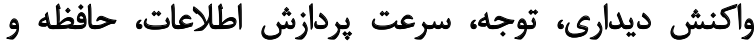

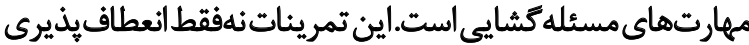

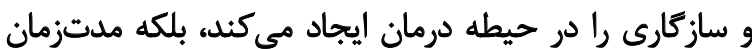

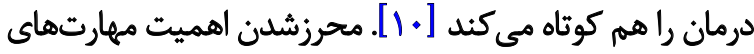

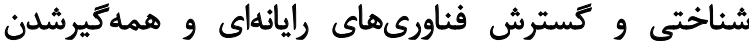

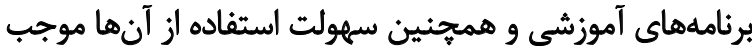

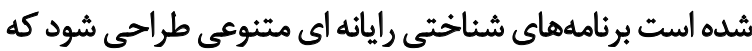

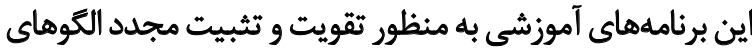

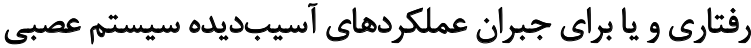

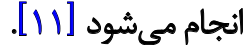

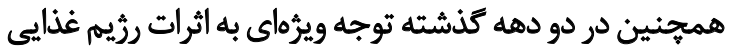

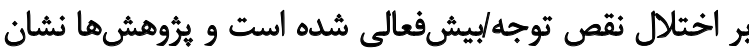

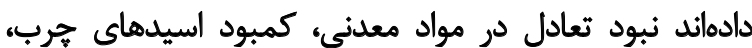

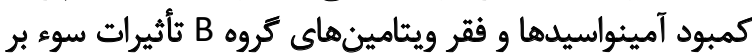
كاركردهاى شناختى دارند [ [ 1 ]]

بر اساس مطالب ذكرشده درباره اهميت و وييامدهاي نقص

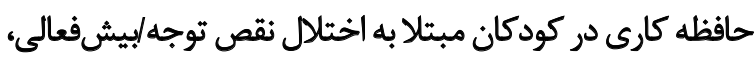

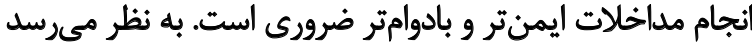
مكملهاى غذايى بيشنياز تشكيل و كاركرد سالم سلولهن بادي

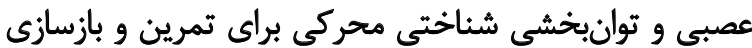

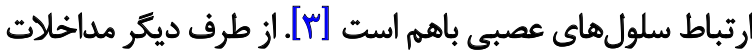

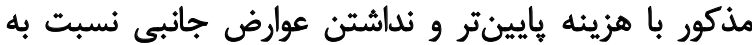

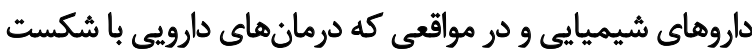

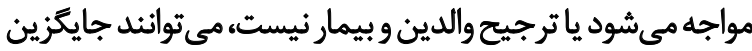

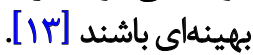
با توجه به كمبود يروهشهايى كه اثربخشى روش هاى درمانى

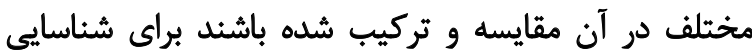

بيشبينى كنئده مشكلاتى هون روان خستشكى، مشكلات

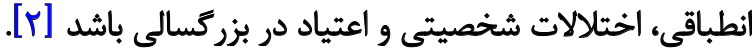
اختلال نقص توجه/بيشفعالى در يُجمين راهنماي

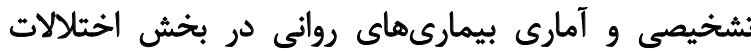

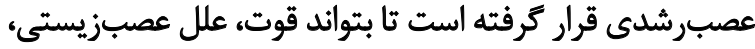

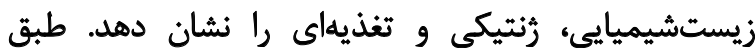

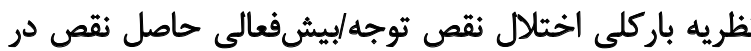

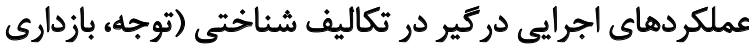

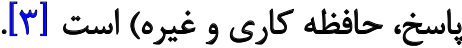
حافظه كارى به عنوان بستر ديكر فعاليتهاى شناختى كاهى

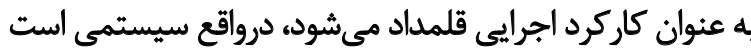

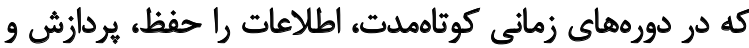

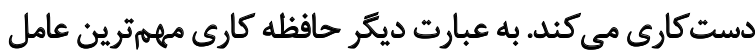

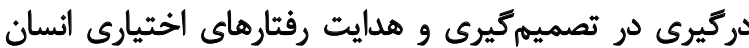

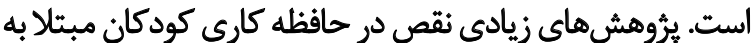

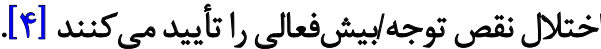
ارجمندنيا و نامجو نيز تزارش كردهاند كه كودكان مبتلا به توريه

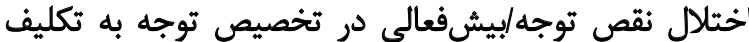

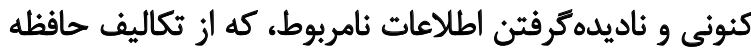

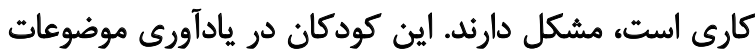

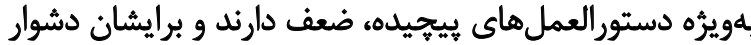

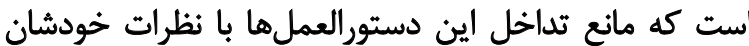

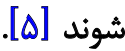

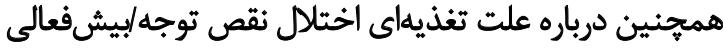

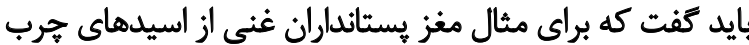

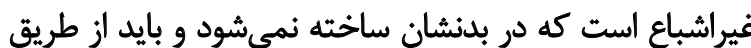

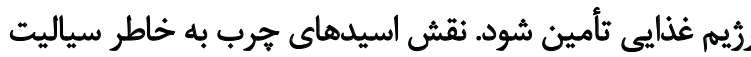

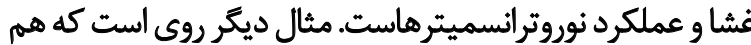

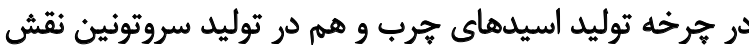

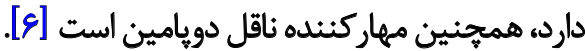

درمانهاى كوناكونى براى اختلال نقص توجه/بيشفعالى

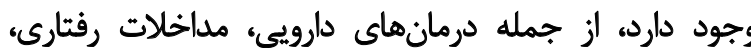

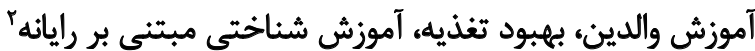

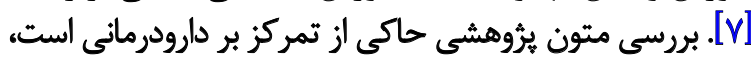

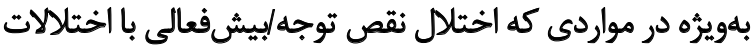

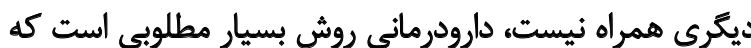

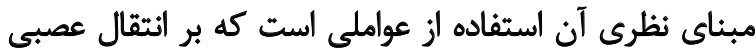

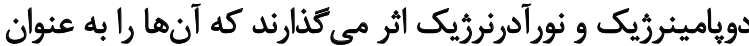

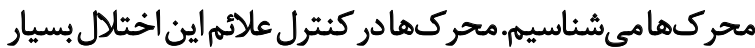

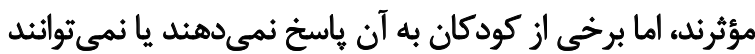


نقص توجه/بيشفعالى، قرارداشتن در دامنه سنى V تأ ال السال،

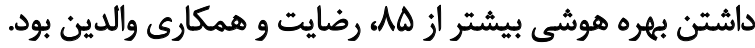

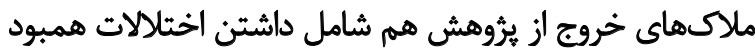

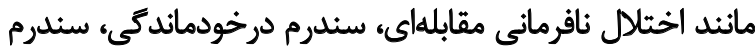

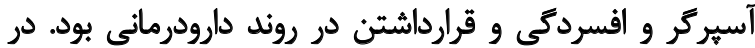

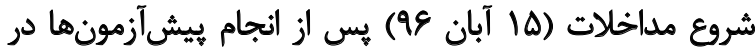

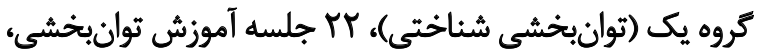

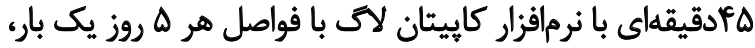

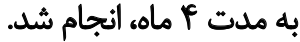
اين تمرينات به صورت تكاليف ديدارى، شنيدارى و ادراكى

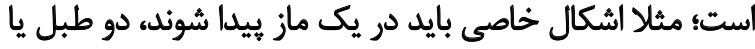

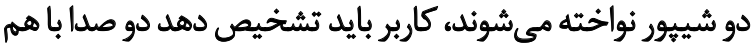

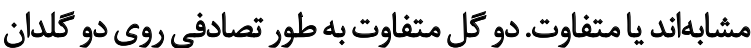

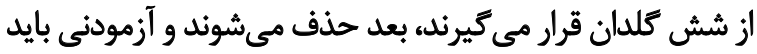

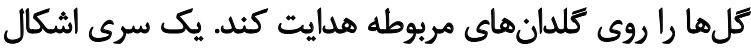

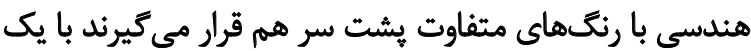

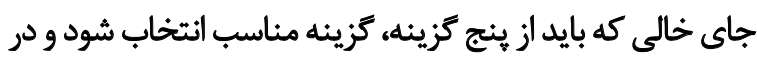
ادامه، تكاليف مشابه با رعايت ترتيب خواستهشيده، انجام مي بـشود. در كروه دو (مداخله مكملهاي غذاييى، يس از انجام

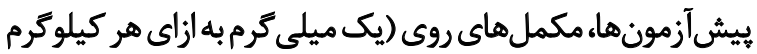

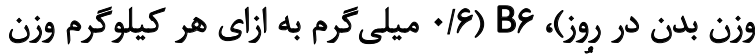

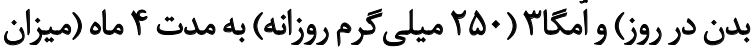

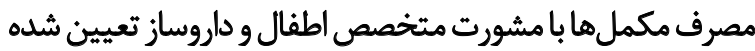

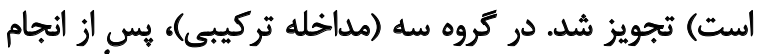

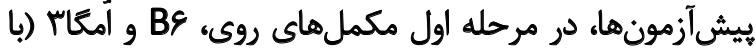

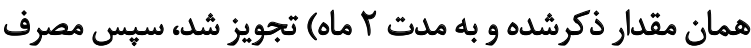

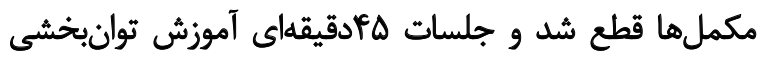

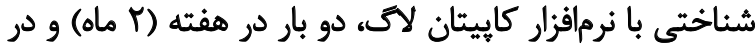

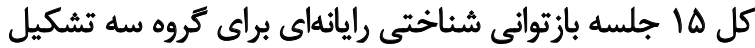

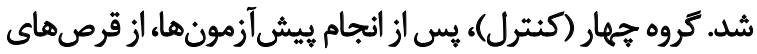

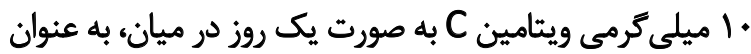

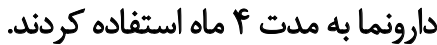

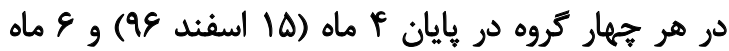

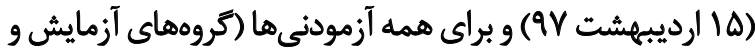

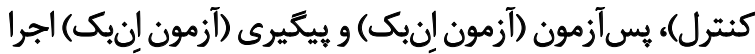

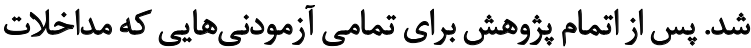

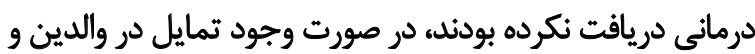

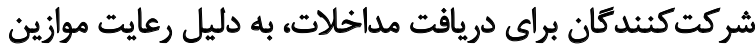
اخلاقى، آموزش كاييتان لاك و مكمل هاى غذائى ماتى ارائه شد.

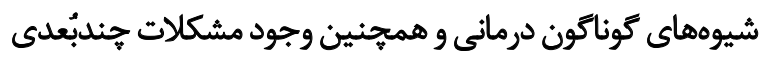

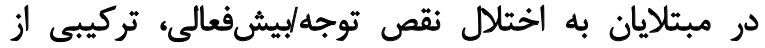

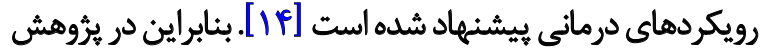

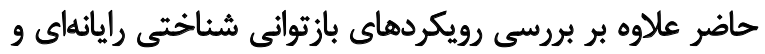

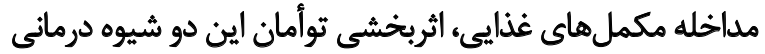

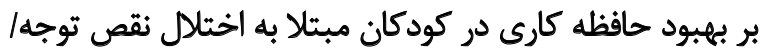
بيش فعالى بررسى شد.

مواد وروش

اين يُووهش، كاربردى است و در آن از طرحى آزمايشى باتروه

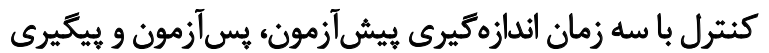

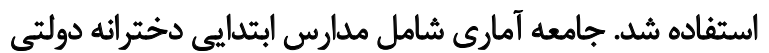

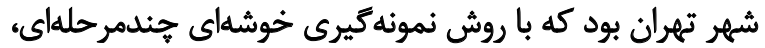

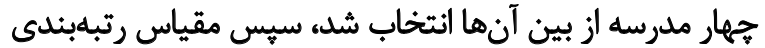

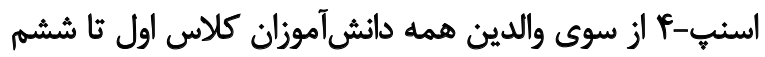

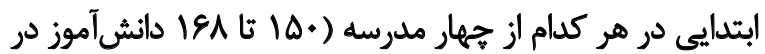

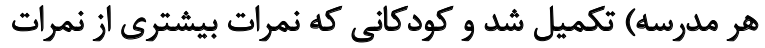

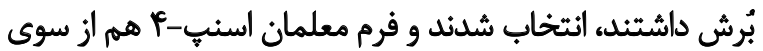
معلمان آنها تكميل و ارزيابى شد.

كر صورت تأييد وجود اختلال هم در خانه و هم در مدرسه اين

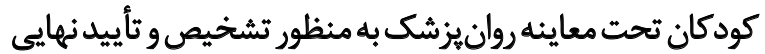

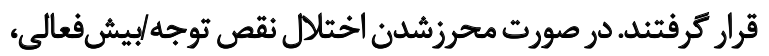

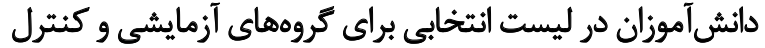

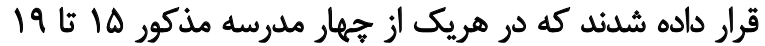

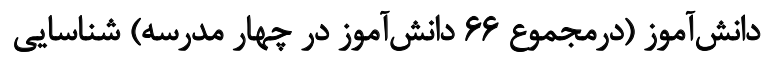

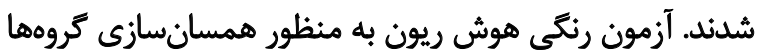

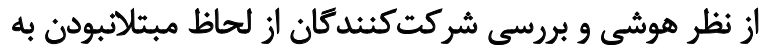

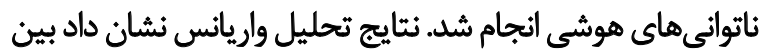

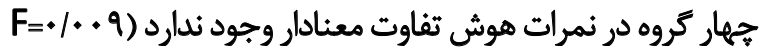
$\left(P>\cdot 1 \cdot \Delta_{6}\right.$

در مرحله بعد مقياس علائم مرضى (فرم والدين) به منظور

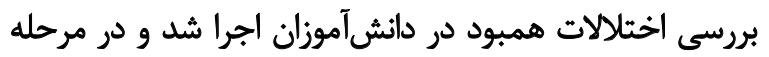

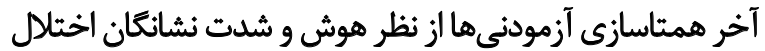

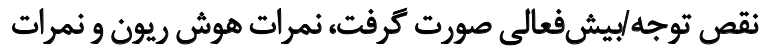

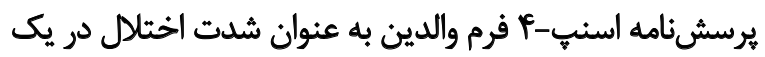

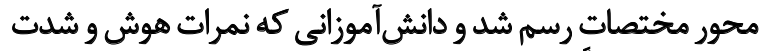

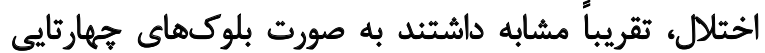

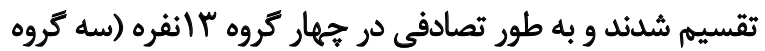

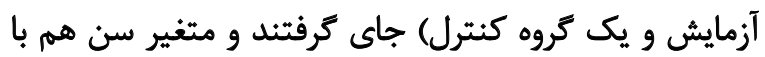

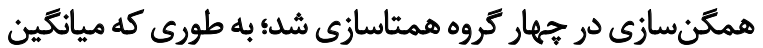
سن جهار كروه تفاوت آمارى معنادارى نداشت

ملاكهاى ورود به يزوهش شامل احراز تشخيص اختلال 
صورت متوالى روى صفحه مانيتور ظاهر مى شود و آزمودنى بايد در

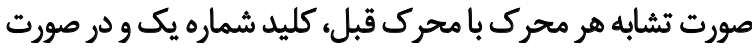

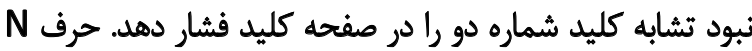

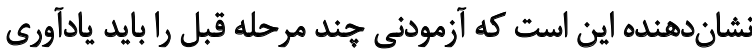

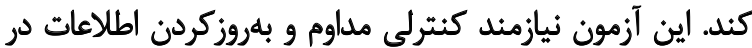

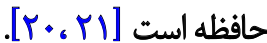

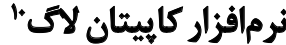

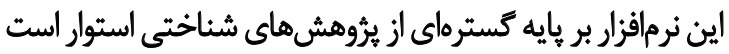

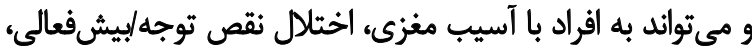

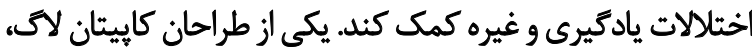

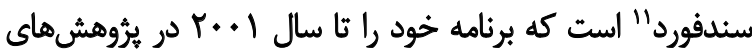

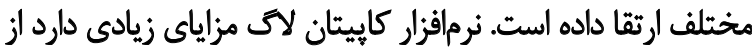

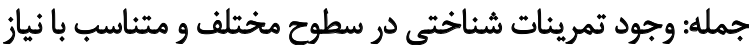

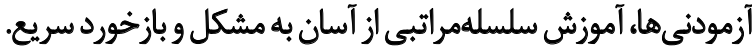

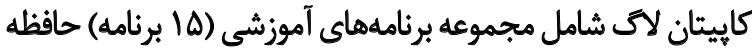

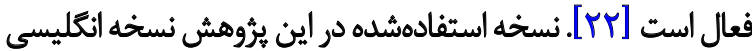

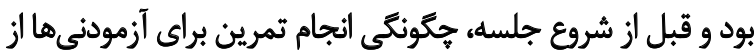
سوى مجرى طرح توضيح داده شد.

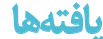

طبق اطلاعات جمعيتشناختى، دامنه سنى آزمودنىها ل آنا

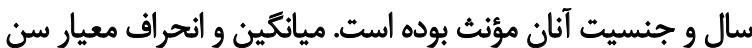

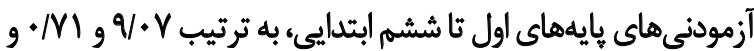

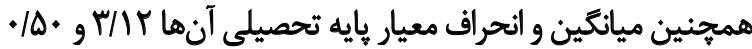

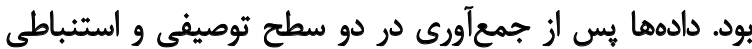

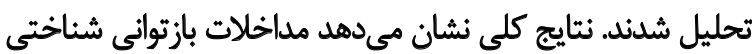

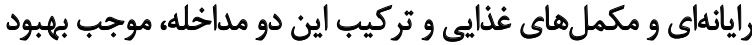

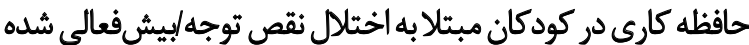

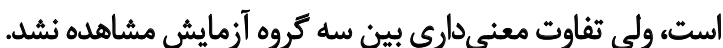

يافتهها نشان مىدهد خردمدقياسهاى حافظه كارى از لحاظ

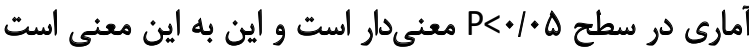

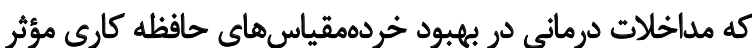

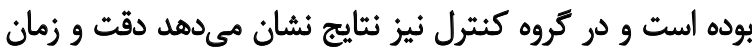

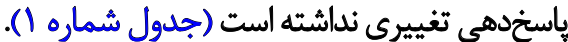

براى آزمون فرضيه اين يثروهش، از روش تحليل واريانس

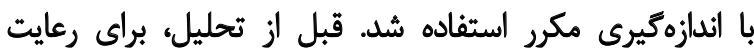

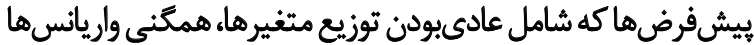

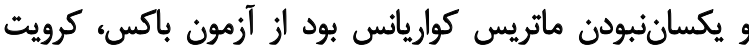

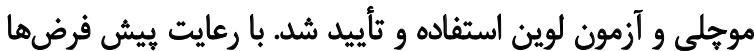

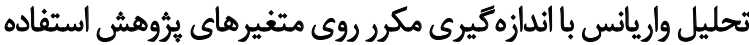

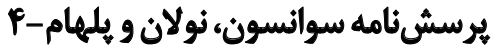

اين مقياس به كوشش سوانسون، نولان و يلهام" براى توصيف

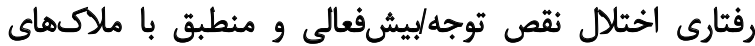

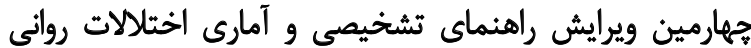

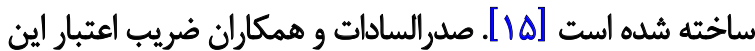

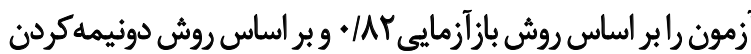

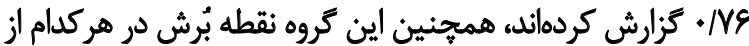

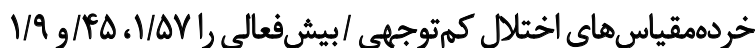

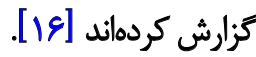

\section{برسشنامه علاثم مرضى كودكانه}

اين برسشنامه لو سؤال دارد و اولينبار اسيرافكين و كادو"

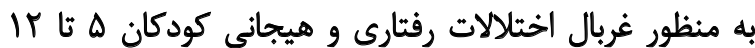

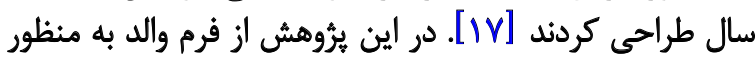

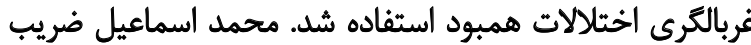

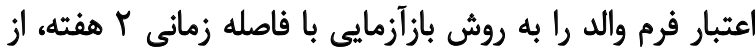

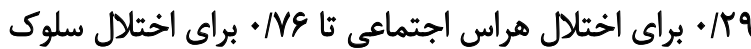

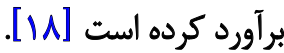

\section{آزمون ماتريس ييشرونده رنكى ريون}

فرم عاتصويرى آزمون هوش ريون را كه بيشتر تصاوير آن رنكى

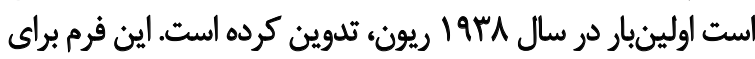

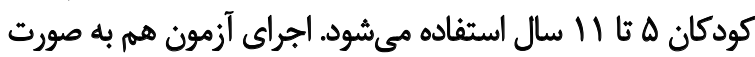

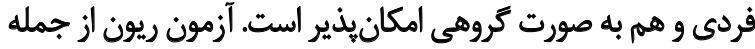

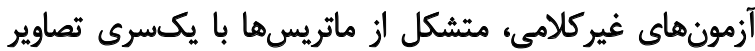

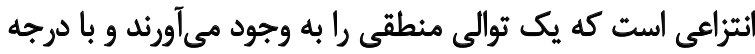

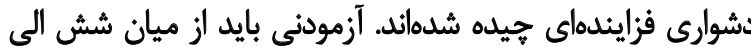

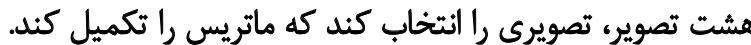

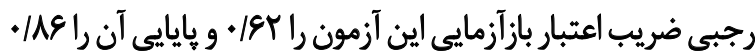

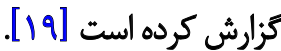

\section{تكليف جند محرك بيشين (انبك)^^}

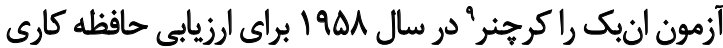

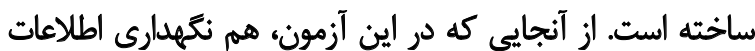

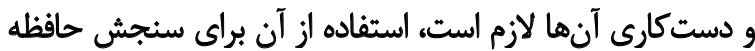

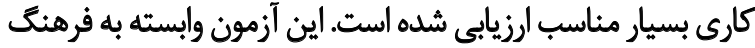

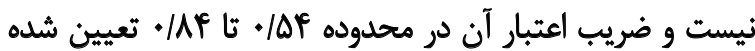

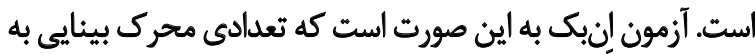

\section{Swanson, Nolan, and Pelham}

5. Children Symptom Inventory-4 (CSI-4)

6. Spirafkin and Gadoo

7. Raven's Colourde Progressive Matrices

8. N-Back

9. Kirchner 
جدول (. ميانكينه انحراف استاندارد، بيشترين و كمترين نمره خردهمقياس هاى حافظه كارى در كروهاى آزمايش و كنترل (n

\begin{tabular}{|c|c|c|c|c|c|c|}
\hline كمترين & بيشترين & انحراف استثاندارد & مراحل & متغيرها & كروه & \\
\hline ח & $A r$ & $E q / 1 Y \pm F / T \Delta$ & يشي أزمون & & \multirow{6}{*}{ 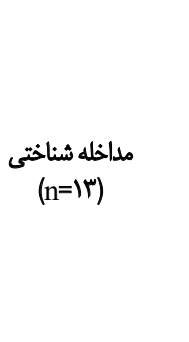 } & \multirow{24}{*}{ آزمايش } \\
\hline A. & 1.0 & QV/F $\pm \mathcal{E V / A V ~}$ & يس أزمون & دقت & & \\
\hline$v$ & 1.0 & $9 . / 9 \pm 8 / \Delta V$ & ييكيرى & & & \\
\hline$\Delta F A$ & 1.89 & $V \cdot V / N$ rTE $\pm Y / g F$ & ييش آزمون & & & \\
\hline DIV & AYA & $9 \Delta q / 1 \cdot Y \pm V / q Y$ & يسآزمون & زمان هاسخدهى & & \\
\hline per & 19v & FE./NQIEYNQA & ييكيرى & & & \\
\hline$\pi$ & 90 & $\Delta \Delta / Y \pm \Delta T / T \Delta$ & ييش آزمون & & \multirow{6}{*}{ 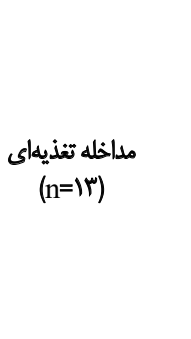 } & \\
\hline$\Delta v$ & 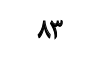 & $S V / N \pm T r / A 1$ & يسآزمون & دقت & & \\
\hline$\Leftrightarrow$ & & VNG $\pm 91 / A 1$ & ييكيرى & & & \\
\hline raI & 801 & DPV/WEEP/PI & ييش آزمون & & & \\
\hline re & 0.1 & $F Q S / T Y \pm T H / T \Lambda$ & يسآزمون & زمان ياسخدهى & & \\
\hline ral & $n \pi$ & $1+ \pm P Y / T \Delta$ & ييكيرى & & & \\
\hline ev & $8 \wedge$ & $\Delta V / E \pm I \Delta / A P$ & ييش أزمون & & \multirow{6}{*}{$\begin{array}{c}\text { مانلله تركيبى } \\
(n=11)\end{array}$} & \\
\hline ex & $M$ & $\eta / \varepsilon \pm 1 \otimes / \& A$ & يس آزمون & قتت & & \\
\hline ve & 95 & $A T / E \pm A F / F \Delta$ & ييكيرى & & & \\
\hline MTI & 819 & $\Delta F T / R T \pm T / A r$ & ييش آزمون & & & \\
\hline$r M$ & $\Delta \Delta r$ & FNAF士EQ/DI & يس آزمون & زمان ياسخدهى & & \\
\hline rape & $\Delta \cdot \Lambda$ & RTNYEDENTY & ييكيرى & & & \\
\hline$\pi$ & vo & 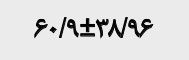 & ييش آزمون & & \multirow{6}{*}{$\underset{(n=11)}{\substack{\text { Sثترل } \\
(n)}}$} & \\
\hline$p$ & va & $8.11 . \pm 9 / \Delta A$ & يسأزمون & قدت & & \\
\hline af & \&V & $\Delta Q / f \pm \pi / m$ & ييكيرى & & & \\
\hline Pr & sn & $\Delta V / q T \pm r / r q$ & ييشآزمون & & & \\
\hline 150 & VFA & $\lambda \cdot \pm \Delta N / \Delta r$ & يس أزمون & زمان ياسخدهى & & \\
\hline are & IfIX & $1.1 T / T H T \pm T N \Delta Q$ & ييكيرى & & & \\
\hline
\end{tabular}

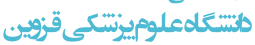

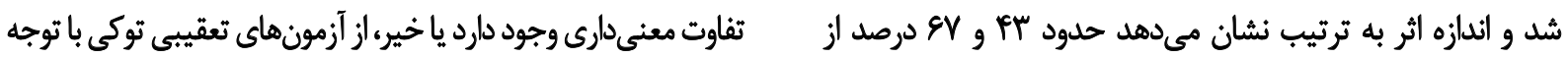

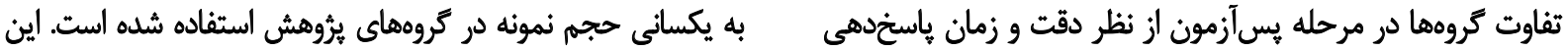

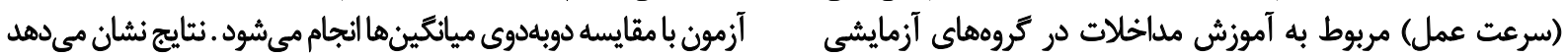

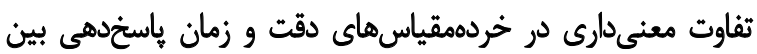

است (جدول شماره (Y).

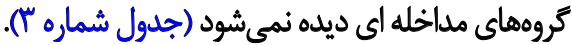

به منظور مشخصكردن اينكه بين اثربخشى روشهاى مداخلهاى

جدول T. نتايج تحليل واريانس با اندازميرى مكرر مربوط به اثر مداخلات درمانى ×زمان آزمون بر متغيرهاى يُروهش

\begin{tabular}{|c|c|c|c|c|c|c|c|}
\hline توان آزمون & اندازه اثر & $\mathbf{P}$ & $\mathbf{F}$ & مياتكين مجذورات & درجه آزادى & مجموع مجذورات & متغير \\
\hline 1 &.$/ 4 r q$ & $+1++\infty 1$ & $\mid r / * 1$ & $Q T+/ \Delta D$ & $r$ & $r n T r / r r$ & دقت \\
\hline 1 & .188 & $+1+\ldots+1$ & $r y /+V$ & PTrTFo/Dq & r & $\mid V+\Delta s+9 / \pi \Delta$ & زعان ياسخدهى \\
\hline
\end{tabular}


جدول "ا. نتايج آزمون تعقيبى خردمقياس هاى حافظه كارى در كروههاى يُروهش

\begin{tabular}{|c|c|c|c|c|c|c|}
\hline كراثه بالا & كراثه هايين & سطح معنى دارى & خطاى استاندارد & تفاوت ميانكين & كروهاهـ & مثغير \\
\hline$T r / q \Delta$ & $11 / F A$ & $+(+\infty)^{*}$ & $r / \pi r$ & WTY & مداخله شناختى در برابرمكملها & \multirow{6}{*}{ دقت } \\
\hline $19 / 8$. & glat & $.1+.1^{*}$ & $r / r i$ & $1 T / . r$ & مداخله شناختى در برابر تركيبي & \\
\hline Te/re & $r . / 91$ & $*(*+)^{*}$ & $r / m$ & $r V / \Delta q$ & مداخله شناختى در برابركنترل & \\
\hline$r / N$ & IV/AV & $.10 .1^{*}$ & $T M$ & Q/re & مها|خله مكمل ها در برابر كتثرل & \\
\hline$-1 \cdot 1 \Delta 4$ & .119 & $.1 . \Delta A$ & T/EV & $-\Delta / 19$ & مداخله مكملها در برابر تركييى & \\
\hline$-9 / .8$ & $-r+1 . \varphi$ & $.1 . . .1^{*}$ & $T / M$ & $\| f / \Delta s$ & مداخله تركيبي در برابر كتترل & \\
\hline $10 V / \Delta V$ & $\Delta T / .8$ & $.1 . . .1^{*}$ & $r \cdot / 4 T$ & $\mid 1 f / m$ & هداخله شناختى در برابرمكملهـا & \multirow{6}{*}{ (سرعت عمل) ياسخى } \\
\hline$I V \cdot / R \Delta$ & $\Delta T / \Delta)$ & $\left..1 \ldots\right|^{*}$ & PQTV & $111 / \pi$ & مداخله شناختى در برابر تركييى & \\
\hline$P q \cdot \Delta$ & $-r / \pi r$ &.$N$ & $r+/ r$ & $-11 / 89$ & مداخله شناختى در برابركتترل & \\
\hline$-I V g / I r$ & $-Y \Delta / M$ & $.1 \% .1^{*}$ & $r$ rq. & -178 & ملاخله مكملها در برابر كتترل & \\
\hline$-\Delta 1 / 9$. & זוIו & .19 .8 & te/To & $-T / M$ & مداخله مكملها در برابر تركييى & \\
\hline$-\ln / / 1 f$ & $-n / 1$ & $.1 . .1^{*}$ & re/Aq & $-I T / I T$ & مداخله تركييى در برابر كنترل & \\
\hline
\end{tabular}

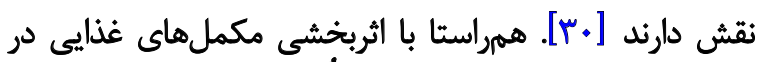

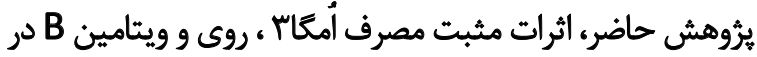

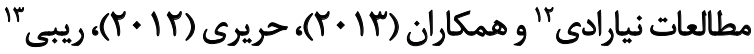

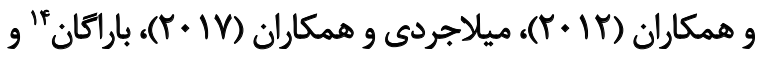

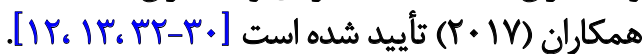
نتايج مداخله تركيبى (توانبخشى شناختى همراه با تجويز

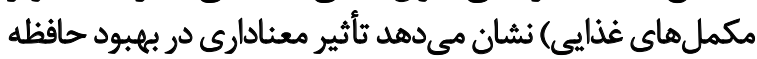

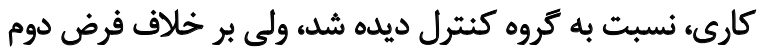

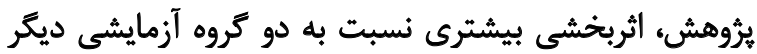

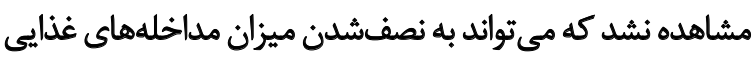

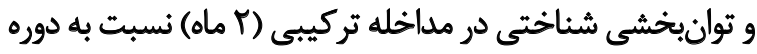

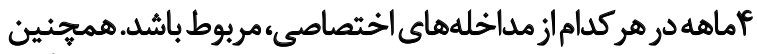

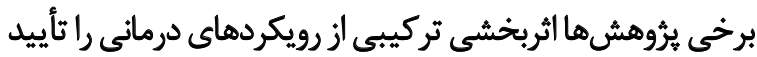

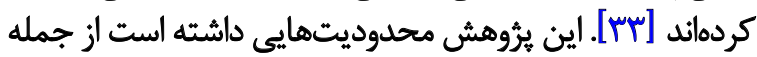

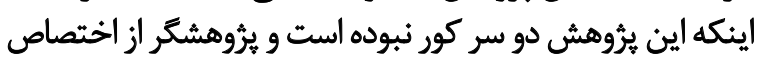

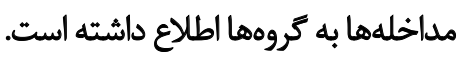

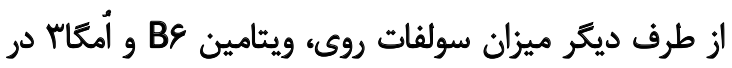

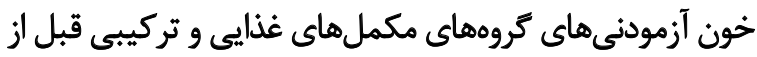

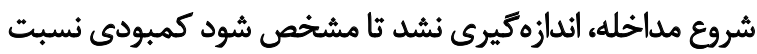

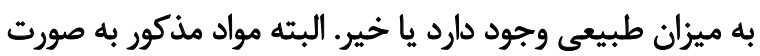

\section{Nyaradi}

13. Riby

14. Barragan

\section{بحث و نتيجه}

هدف از اين يثوهش بررسى تأثير آموزش شناختى مبتنى بر

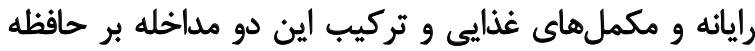

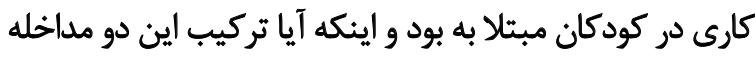

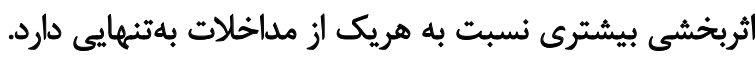

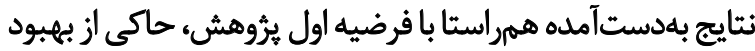

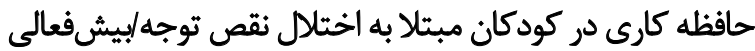

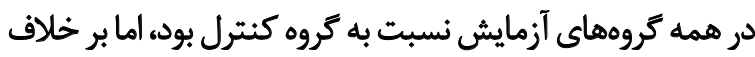

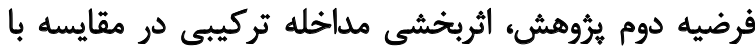

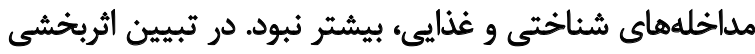

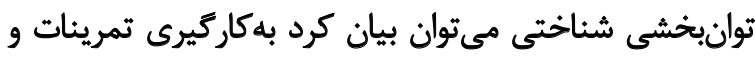

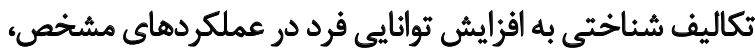

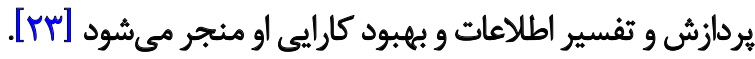
به عبارتى بازتوانى شناختى، به بهبود توانايىهاي شناختي و وتئي

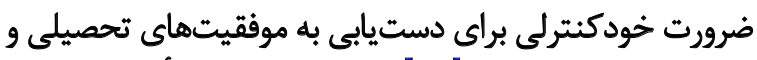

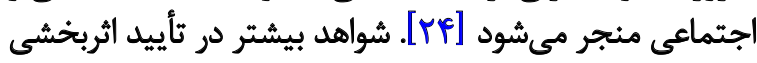

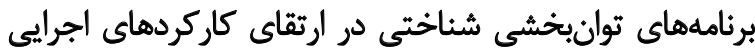

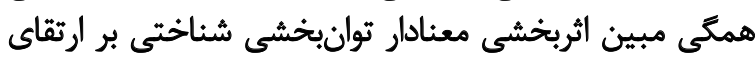

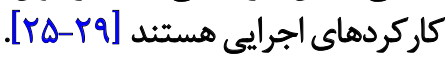

در تبيين اثربخشى مكملهاى غذايى مى توان بيان كرد كه

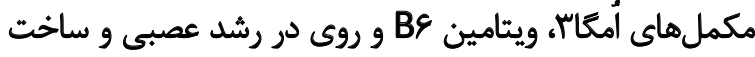

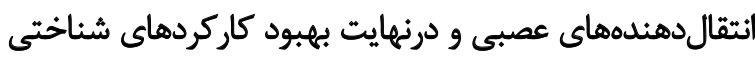




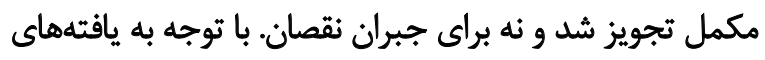

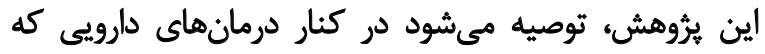

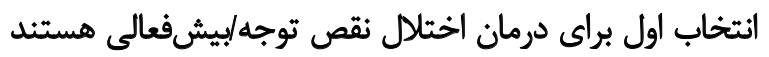

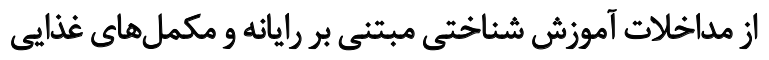

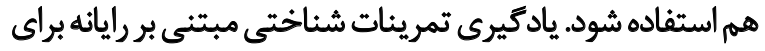

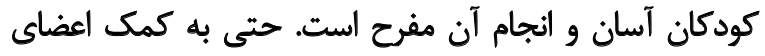

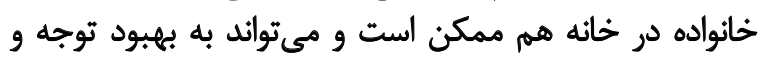

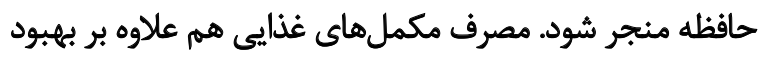

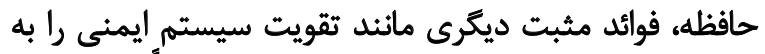

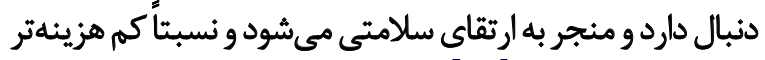

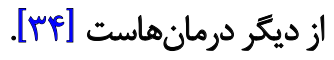

معىتوان هر دو مداخله را با توجه به مزاياى ذكرشده و نداشتن

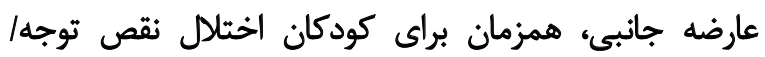

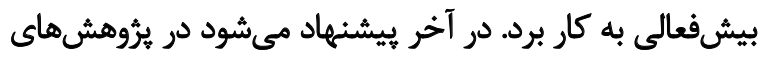

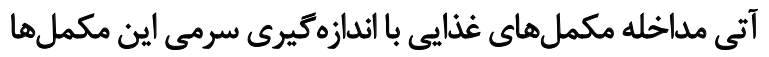

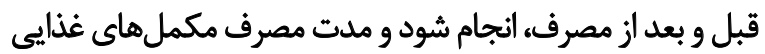

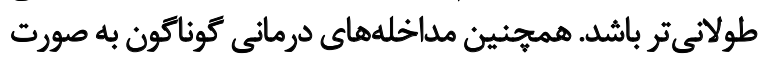

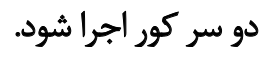

مالامظات أخالاقي

بيروى ازٔ اصول اخلاق بثوهش كميته اخلاق دانشعاه علوم بزشكى سمنان اين مطالعه را تأييد و با كد IR.SEMUMS.REC.1397.008 ثبت كرده است.

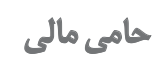

اين مقاله از يايانثامه دكتراي نويسنده اول در كروه روانشناسي

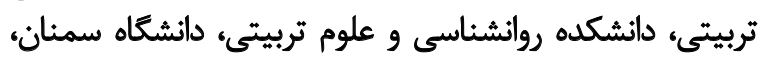

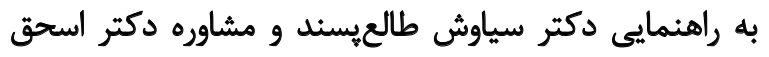
رحيميان بوكر كرفته شده است.

$$
\text { مشاركت نويسندكّان }
$$

تمام نويسندكان در طراحي، اجرا و نكارش همه بخشهائ

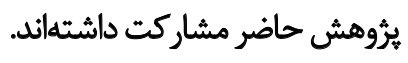

$$
\text { ثقارض مناقع }
$$

بنابر اظهار نويسندكان، اين مقاله تعارض منافع ندارد. 


\section{References}

[1] Schulz-Zhecheva Y, Voelkle M, Beauducel A, Buch N, Fleischhaker $C$, Bender $S$, et al. ADHD traits in German schoolaged children: Validation of the German strengths and weaknesses of ADHS symptoms and normal behavior (SWAN-DE) scale. J Atten Disord. 2017:1087054716676365. [DOI:10.1177/1087054716676365]

[2] Caye A, Swanson J, Thapar A, Sibley M, Arseneault L, Hechtman $L$, et al. Life span studies of ADHD- conceptual challenges and predictors of persistence and outcome. Curr Psychiatry Rep. 2016; 18(12):111. [DOI:10.1007/s11920-016-0750-x] [PMID] [PMCID]

[3] Barkley RA. Attentiondeficit hyperactivity disorder: A handbook for diagnosis and treatment. New York: Guilford Press; 2015.

[4] Bunford N, Evans Sw, Langberg JM. Emotion dysregulation is associated with social impairment among young adolescents with ADHD. J Atten Disord. 2018; 22(1):66-82. [DOI:10.1177/1087054714527793] [PMID]

[5] Arjmandnia A, Namjoo S. Efficacy of Memory Specificity Training (MEST) on underlying mechanisms of Over General autobiograpical Memory (OGM) in people with major depression and childhood traumatic experience. Isfahan Univ Med Sci. 2015; 33(329):442-56. [In Persian]

[6] Lahouti M, Zavoshy R, Noroozi M, Rashidkhani B, Rostami R. Association between dietary patterns and depressive symptoms in adult women living in Tehran. J Qazvin Univ Med Sci. 2015; 19(4):32-41. [In Persian]

[7] Saadat M. Which symptoms of ADHD would Improve with play therapy and Yoga? J Exceptional Children. 2011; 11(1):45-56. [In Persian]

[8] Dobson-Patterson R, O'Gorman JG, Chan RC, Shum DH. ADHD subtypes and neuropsychological performance in an adult sample. Res Dev Disab. 2016; 55:55-63. [DOI:10.1016/j. ridd.2016.03.013]

[9] Fitzgerald M, Bellgrove M, Gill M. Handbook of Attention Deficit Hyperactivity Disorder. Hoboken: John Wiley \& Sons; 2007.

[10] Noukani M. Cognitive rehabilitation using computers to reduce attention deficit disorder (ADHD) [PhD. dissertation]. Tehran: University of Rehabilitation sciences and social welfare; 2010. [In Persian]

[11] Hutton B, Tetzlaff J, Yazdi F, Thielman J, Kanji S, Fergusson D, et al. Comparative effectiveness of monotherapies and combination therapies for patients with hypertension: Protocol for a systematic review with network meta-analyses. Syst Rev. 2013; 2:44. [DOI:10.1186/2046-4053-2-44] [PMID] [PMCID]

[12] Riby L, Smith M, Foster J. Nutrition and mental performance: A lifespan perspective. London: Macmillan International Higher Education; 2012. [DOI:10.1007/978-1-137-00689-9]

[13] Barragán E, Breuer D, Döpfner M. Efficacy and safety of omega-3/6 fatty acids, methylphenidate, and a combined treatment in children with ADHD. J Atten Disord. 2017; 21(5):43341.
[14] Millichap JG, Yee MM. The diet factor in attention deficit/hyperactivity disorder. Pediatrics, 2012; 129(2):330-7. [DOI:10.1542/peds.2011-2199] [PMID]

[15] Cortese S, Ferrin M, Brandeis D, Holtmann M, Aggensteiner P, Daley D, et al. Neurofeedback for attention-deficit/hyperactivity disorder: Meta-analysis of clinical and neuropsychological outcomes from randomized controlled trials. J Am Acad Child Adolesc Psychiatry. 2016; 55(6):444-55. [DOI:10.1016/j. jaac.2016.03.007] [PMID]

[16] Sadrolsadat S, Hoshyary Z, Zamani R, Sadrolsadat L. Determinatio of psychometrics index of SNAP-IV rating scale in parents execution. Arch Rehab. 2008; 8(3):59-65. [In Persian]

[17] Hathaway WL, Barkley RA. Self regulation ADHD and child religiousness. J Psych Christ. 2003; 22(2):101-14.

[18] Mohamadesmaiel E, Alipour A. A preliminary study on the reliability, validity and cut off points of the disorders of Children Symptom Inventory-4 (CSI-4). J Excep Child. 2002; 2(3):239-54. [In Persian]

[19] Rajabi G. Normalizing The raven coloure progressive matrices test on students of city Ahvaz. Contemp Psychol. 2008; 3(1):23-32. [In Persian]

[20] Jaeggi SM, Studer-Luethi B, Buschkuehl M, Su YF, Jonides J, Perrig WJ. The relationship between $\mathrm{N}$-back performance and matrix reasoning-implications for training and transfer. Intelligence. 2010; 38(6):625-35. [DOI:10.1016/j.intell.2010.09.001]

[21] Kane MJ, Conway AR, Miura TK, Colflesh GJ. Working Memory, attention control, and the N-back task: A question of construct validity. J Exp Psychol: Learn Mem Cog. 2007; 33(3):61522. [DOI:10.1037/0278-7393.33.3.615] [PMID]

[22] Cortese S, Ferrin M, Brandeis D, Buitelaar J, Daley D, Dittmann RW, et al. Cognitive training for attention-deficit/hyperactivity disorder: Meta-analysis of clinical and neuropsychological outcomes from randomized controlled trials. J Am Acad Child Adolesc Psychiatry. 2015; 54(3):164-74. [DOI:10.1016/j. jaac.2014.12.010] [PMID] [PMCID]

[23] Saha P, Chakraborty P, Mukhopadhyay P, Bandhopadhyay D, Ghosh S. Computer based attention training for treating a child with attention deficit/hyperactivitydisorder: An adjunct to pharmacotherapy- A case report. J Pharm Res. 2015; 9(11):612-7.

[24] Javadi TH, Borjali M, Borjali A. Effectiveness of Barkley's behavioral parent training in reducing symptoms of children with Attention Deficit Hyperactivity Disorder. J Qazvin Univ Med Sci. 2014; 17(6):47-52. [In Persian]

[25] Sohrabi f. Effectiveness of Computer-Asisted Cognitive Remediation (CACR) and psycho simulant drugs on clinical symptoms of children with Attention Deficit/Hyperactivity Disorder (ADHD). Contemp Psychol. 2012; 7(2):51-60. [In Persian]

[26] Rostaman H, Talepasand s, Nazifi M, The effect of executive function training on executive performance and behavioral symptoms of children with Attention Deficit Hyperactivity Disorder. J Clin Psych. 2013; 5(1):93-106. [In Persian]

[27] Steiner NJ, Sheldrick RC, Gotthelf D, Perrin EC. Computerbased attention training in the schools for children with atten- 
tion deficit/hyperactivity disorder: a preliminary trial. Clin Pediatr. 2011; 50(7):615-22.[DOI:10.1177/0009922810397887] [PMID]

[28] Abdi A, Arabani DA, Hatami J, Parand A. The effect of cognitive computer games on Working Memory, attention and cognitive flexibility in students with attention deficit/hyperactivity disorder. J Excep child. 2014; 14(1):19-33. [In Persian]

[29] Najarzadegan M, Nejati V, Amiri N. Effect of cognitive rehabilitation of Working Memory in reducing behavioral symptoms (attention deficit and impulsivity) of children with attention deficit and hyperactivity disorder. Neuropsychol. 2015; 1(1):52-65. [In Persian]

[30] Nyaradi A, Li J, Hickling S, Foster J, Oddy WH. The role of nutrition in children's neurocognitive development, from pregnancy through childhood. Front Human Neurosci. 2013; 7(97):1-16. [DOI:10.3389/fnhum.2013.00097]

[31] Hariri M, Djazayery A, Djalali M, Saedisomeolia A, Rahimi A, Abdolahian E. Effect of $n-3$ supplementation on hyperactivity, oxidative stress and inflammatory mediators in children with attention-deficit-hyperactivity disorder. Malays J Nutr. 2012; 18(3):329-35. [PMID]

[32] Milajerdi AR, Mousavi SM, Hassanzadeh Keshteli A, Esmaillzadeh A, Roohafza HR, Afshar $\mathrm{H}$, et al. The relation between breakfast consumption and psychological symptoms among adults. J Qazvin Univ Med Sci. 2017; 21(3):66-75. [In Persian]

[33] World Medical Association. World Medical Association Declaration of Helsinki: Ethical principles for medical research involving human subjects. JAMA. 2013; 310(20):2191-4. [DOI:10.1001/jama.2013.281053] [PMID]

[34] Farhud D, Shalileh M. Relation between omega 3 fatty acid, iron, zinc and treatment of ADHD. Zahedan J Res Med Sci. 2014; 16(8):1-5. 